\title{
Biocatalytic intramolecular cyclopropanation
}

J. Am. Chem. Soc. https://go.nature.com/2WfzaKt (2019).

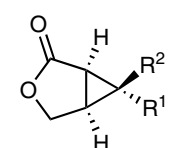

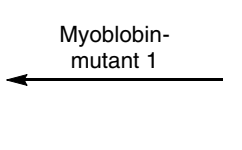

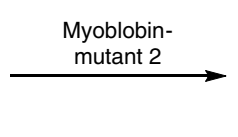

mutant 2

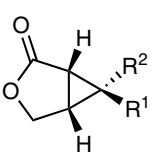

$\mathrm{R}^{1}=$ aromatic, aliphatic; $\mathrm{R}^{2}=\mathrm{Me}, \mathrm{H}$

Cyclopropane-fused $\gamma$-lactones are found in many bioactive molecules and intermediates for the synthesis of valuable compounds. However, biocatalytic in vitro strategies for their one-step production from simple starting materials remained elusive.

Now, Rudi Fasan and colleagues report the biocatalytic intramolecular cyclopropanation of allyl diazoacetate substrates to cyclopropyl- $\boldsymbol{\gamma}$-lactones, extending their previous research on enzymatic intermolecular cyclopropanation. The current work was spurred by the discovery that haemin and wild-type haemproteins show native activity in the intramolecular cyclopropanation of cinnamyl 2-diazoacetate, leading to the corresponding bicyclic product.

Among the tested proteins, myoglobin (Mb) appeared to be most promising, although the reactivity was modest. Therefore, the authors designed and screened small libraries of Mb-mutants and identified variants with improved activity and enantioselectivity in the production of $(1 R, 5 S, 6 S)$-configured cyclopropane-fused $\gamma$-lactones, enabling up to $99 \%$ conversion and over $99 \%$ enantiomeric excess.

Derivatives of cinnamyl 2-diazoacetate carrying para-, meta- and orthosubstituents, as well as both electron withdrawing and electron donating groups on the phenyl moiety, were well tolerated. Furthermore, diazoacetates equipped with either a sterically demanding naphtyl moiety, unactivated olefins or multiple olefinic groups were also accepted by the Mb-mutants.

Subsequently, the authors designed another library to screen for a stereocomplementary $\mathrm{Mb}$-variant to catalyse the same reaction with $(1 S, 5 R, 6 R)$ instead of $(1 R, 5 S, 6 S)$-selectivity. Indeed, a variant with opposite enantioselectivity was identified that - for a similar set of substrates - enabled conversions and enantiomeric excess of up to $99 \%$ and $96 \%$, respectively. A stereochemical model was proposed, helping to rationalize the effect of the mutations on the enantioselectivity.

Although the turnover numbers in this work are rather moderate, this research paves the way for the use of haemproteinbased catalysts in intramolecular cyclopropanation reactions giving access to valuable compounds. The high enantioselectivity, stereodivergent selectivity and rather broad substrate scope are worthwhile for synthetic chemistry. In light that only a relatively small number of $\mathrm{Mb}$-variants were screened, it can be anticipated that further engineering efforts in the near term will make biocatalytic intramolecular cyclopropanation a truly powerful synthetic tool.

Jan-Stefan Völler

Published online: 13 June 2019

https://doi.org/10.1038/s41929-019-0313-8 